agent they contain cannot act effectively. Our experience with this method is not extensive, but we believe that any case of erysipelas may be cut short in its earlier stages by this method, and that the exclusion from the part of putrefactive bacilli contained in the atmosphere will effectively prevent suppuration in the subcutaneous tissues. Mossbaum and Sir Dyce Duckworth use an ointment con. taining ichthyol. Koch recommends the application, by means of a camel hair brush, of a thin layer of an ointment composed of creolin one part, iod joform four parts, lanolin ten parts. The ointment is to be applied for some two or three inches of healthy skin around as well as over the erysipelatous patch, and then all is covered with a thin layer of gutta percha. In twenty-five cases thus treated a fall of temperature occurred, and the skin resumed its normal colour after two or three applications.

Nolte paints the entire surface of the affected part with a mucilage of gum arabic, containing three to five per cent. of phenic acid. Very excellent results were obtained by Rosenbach's method of washing the erysipelatous part and the surri unding skin with soap, then bathing the patch daily with a five per cent. solution of phenic acid, dissolved in absolute alcohol. Millihem has in three cases obtained an immediate and complete jugulation of erysipelas by painting with campho-phenique, two parts to one part olive oil. Hallopeau speaks favourably of the use of a solution of salicylate of sodium (one in twenty), thick cloths being wetted with the solution and applied, these being then covered with a layer of gutta percha or rubber cloth to prevent evaporation. Kingsbury recommends ergotine in equal parts of distilled water. He has found it a painless and almost certain cure. Hunter advocates the injection of carbolic acid in the healthy skin at a distance from the part affected, a method which unfortunately is very painful. Kraske recommends making incisions in the healthy skin around the patch before applying the antiseptic.

It is interesting that erysipelas, like some other specific diseases, has been made to serve as a remedial agent. Babchinski, while attending a very grave case of diphtheria, noticed a remarkable improvement in the patient's condition on the appearance of an erysipelatous rash on the face. This suggested to him the possibility of good results to be obtained by the inoculation of diphtheritic patients /with blood from erysipelatous patients, and in several cases this treatment seemed to produce a cure. More recently he has found the inoculation of cases of diphtheria with cultures of the erysipelas microbe has proved equally satisfactory. We referred in a previous issue (Hospital, Aug. 9, 1890) to Dr. Kleeblatt's obscrvations on the effects of erysipelas in cancer.

\section{COLD TREATMENT OF PNEUMONIA.}

We are still in doubt as to the part played by micro organisms in the production of pneumonia. hoch, Fried. lander, Klebs, and other observers have described specific micrococci, which have been found in the exudation, in the lymphatics of the lungs, and in blister fluid. Further, it has been stated that pneumonia has been produced in rabbits by the injection of cultivations of the preumococcus, and in mice by the inhalation of a spray charged with these germs. The clinical histories of cases of pneumonia so forcibly confirm these views that the fact that similar micro-organisms have been found apart from pneumonia, can only lead us to suppose that we are unable to distinguish between micrococci, which are similar in appearance, but widely different in properties, or that in these latter cases the requisite conditions for the manifestation of their peculiar nature are wanting. Dr. Lipıri, of Polerino, has demonstrated by his experiments the precise part played by cold in the production of pneumonia. $\mathrm{He}$ found that the simple endo-tracheal injection of the sputum of pneumonia, or the injection of the pleuritic exudation of animals which had died from pneumococci, did not produce pneumonia. If, however, the animal, either before the injection or soon after, was exposed to cold, the results were very different. Six out of eight of the animals treated in this way died with pneumonia. The alveoll were found to be infil. trated with exudation as in ordinary lobar pneumonia. Dr. Lipar $i$ is of opinion that the cold acts by paralysing the ciliated epithelium of the bronchi, causing their mucous membrane to swell at the same time. These two conditions would favour the entrance and descent of infectious matter into the alveoli. It is very possible, however, that the pneumococcus requires a low temperature for the development of its specific infective qualities. These researches have a practical bearing on the treatment of pneumonia by ice-bags and cold baths that have been re-introduced recently, a practice adopted in the seventeenth and eighteenth centuries, and more recently by Niemeyer twenty years ago. Dr. Lees has used the ice-bag in pneumonia during the last four years in eighteen cases, without a death, of these, two at least, in his opinion, would have certainly died without the ice-bag, and in two others of these cases would have almost certainly done so. By its use the temperature was lowered in all cases, but he found that the improvement did not consist only on the reduction of temperature. The physical signs were arrested in development, even before this crisis, and rapidly improved subsequently. He has never had any bad results with its use. Dr. Goodhart, too, has had excellent results with the ice-bag. In three out of his eighteen cases, symptoms of collapse ensued, but this was of a temporary nature and easily counteracted by the application of warmth to the feet and the administration of brandy. He warns us against adopting this method in children under two years of age. In hyperpyrexial cases the use of cold baths may save the patient when all other means fail. Dr. Angel Money has advocated the more general use of ice-bags in his treatment of broncho-pneumonia. in children. He has treated many severe cases in this way with success, no matter what the cause of the disease was. The smaller the child the more marked the effects. He has used Leiter's tubes with advantage. The treatment must be carried out systematically in order to obtain any good results. He has found that the duration of the disease is on the whole shortened, and convalescence is almost invariably more rapid. Vomiting is staved off and diarrhœe not increased by the cold in cases in which these complications occur. Albuminuria is not rendered worse nor is otitis media more frequent than in cases treated without cold.

\section{SURGERY.}

\section{TUBERCULAR DISEASES OF THE BONES AND} JOINTS.

The nature of the processes which occur in so-called strumous affections of the joints and the contiguous portions of bone have been recently investigated by König, Baumgarten, Barker, Watson Cheyne, and others. There is an accumulation of evidence to show that these diseases are due essentially to the presence of the bacillus tuberculosis, although these germs may not always be found In his im. portant lectures at the Royal College of Surgeons, Mr. Watson Cheyne srys, "I attach no importance to the demonstration of tubercle bacilli as a means of diagnosing these bone and joint diseases ;" but he considers that there are other characteristics which are more constant and essentially tubercular. He defines a tubercle histologically as "a micro. scopic nodule, generally round or oval in shape, composed of a central portion made up of epithelioid cells, and sometimes giant cells, surrounded by a layer consisting of cells of inflammatory origin, or of more completely formed fibrous tissue." This fibrous ring may or may not.be the remains of 